\title{
Rolling of Flat Aluminum Strips with Tailored Mechanical Properties
}

\author{
Olexandr Grydin ${ }^{1 \mathrm{a}}{ }^{*}$, Serhii Bondarenko ${ }^{2 \mathrm{~b}}$, Mykhailo Stolbchenko ${ }^{1 \mathrm{c}}$, \\ Mirko Schaper ${ }^{1 \mathrm{~d}}$ \\ ${ }^{1}$ Chair of Materials Science, University of Paderborn, Pohlweg 55, 33098 Paderborn, Germany \\ ${ }^{2}$ Chair of Metal Forming, National metallurgical Academy of Ukraine, Gagarin Prospekt 4, 49600 \\ Dnipropetrovsk, Ukraine \\ agrydin@lwk.uni-paderborn.de, bsergey_bondarenko_89@bk.ru, \\ cstolbchenko@lwk.uni-paderborn.de, 'schaper@lwk.uni-paderborn.de
}

Keywords: Strip, tailored properties, flat rolling, strain hardening, aluminum

\begin{abstract}
In this study, the possibility of producing of flat aluminum strips with tailored mechanical properties across their width by means of rolling was examined. The aim of the work was an experimental analysis of the effect of cold rolling of the strips with a pre-profiled crosssection in flat rolls. Because of different work hardening of the various elements of the pre-profiled strips a characteristic profile of mechanical properties over the strips cross-section was created. The performed tensile tests and hardness measurements proved the possibility of setting of tailored properties in flat strips by means of rolling. The difference between the mechanical characteristics of various strip's elements for the selected aluminum alloy can reach $40 \%$.
\end{abstract}

\section{Introduction}

One of the main principles of lightweight structure design is the localized application of materials with different properties such as stiffness, strength, ductility, toughness, etc. The combination of mechanical characteristics can be achieved either by the use of various materials, or by the implementation of a mono-material in different states in order to manufacture diverse construction elements. An economic efficient example of the practical implementation of both of these approaches is the processing of special semi-finished strips with heterogeneous characteristics - the so-called tailored blanks. Tailored blanks are already widely employed in the automotive industry for the production of various components of the car body by means of the sheet metal processing. The most representative construction element of vehicles with tailored characteristics is a B-pillar $[1,2]$.

There are several types of blanks with heterogeneous mechanical properties, which can be classified into three groups depending on their manufacturing principles. Firstly, tailored welded blanks (TWB) represent the most widespread group. These semi-finished parts are produced by butt welding of several strips of different alloys or various thicknesses [3, 4]. Secondly, tailored rolled blanks (TRB) are monometallic strips with a profiled shape, which are obtained by flexible rolling $[3,5]$ or by strip profile rolling $[3,6]$ with subsequent annealing. In this case, the mechanical properties of different elements of a TRB are similar. The heterogeneity concerns solely the shape factor of the strip cross-sections. The tailored mechanical properties of a monometallic material can be created by various heat treatment modes of different regions in one strip. Thirdly, strips of the group are called tailored heat treated blanks (THTB). However, with respect to thermal treatments, one significant limitation can be identified - THTB can solely manufactured with heat-treatable metals and alloys. [3, 7]. At present, most TWB, TRB and THTB are manufactured of different steels as well as of various aluminum alloys. 


\section{Motivation and Objective}

A review of methods on flat feedstock production with tailored mechanical properties revealed that TWB of different alloys and THTB can provide a significant difference in strength characteristics of the material in various areas of a strip. However, subsequent to the welding procedure and during the TWB manufacturing, a local microstructural inhomogeneity can be detected in the welding seam zone as well as in the heat-affected zone. Generally, a normalization of the welding seam structure is often impossible due to the dissimilarity of the applied materials. Thus, the unfavorable microstructure transmits to the final product. THTB can be produced utilizing only hardening materials. In addition, an energy- and cost-intensive heating in a complex heating/cooling system is essential for the local heat treatment.

From this perspective, the objective of this study is an experimental validation on the production of flat strips with tailored mechanical properties of materials which thermally cannot be hardened. Here, the effect of various local cold work hardening of material during rolling of pre-profiled strips may be used.

\section{Experimental Procedure}

The technical purity aluminum with respect to EN AW-1050 was selected as a model material for the experimental investigation of the different deformation hardening modes of pre-profiled strips. The chemical composition of the utilized alloy is shown in Table 1.

Table 1. Chemical composition in weight percent of the investigated aluminum EN AW-1050

\begin{tabular}{|c|c|c|c|c|c|c|c|c|}
\hline $\mathrm{Al}$ & $\mathrm{Fe}$ & $\mathrm{Si}$ & $\mathrm{Zn}$ & $\mathrm{Ti}$ & $\mathrm{Mg}$ & $\mathrm{Mn}$ & $\mathrm{Cu}$ & Balance \\
\hline 99.5 & 0.40 & 0.25 & 0.07 & 0.05 & 0.05 & 0.05 & 0.05 & 0.03 \\
\hline
\end{tabular}

The initial strips the dimensions of $420 \mathrm{~mm} \times 180 \mathrm{~mm} \times 2.9 \mathrm{~mm}$ were used to conduct the experiments. An $80 \mathrm{~mm}$ wide steel strip has been placed unidirectional in the center of the initial aluminum sample (Fig. 1a). Two steel strips with a thickness of $2.00 \mathrm{~mm}$ for the first series of experiments, and the thickness of $0.55 \mathrm{~mm}$ for the second series of tests were used. The steel strip length was equal to the length of the initial aluminum sample. The interface surface of the steel strips was coated with a graphite suspension in order to avoid sticking between both materials. These steel-aluminum sandwiches were flat rolled in the longitudinal direction for several passes at the room temperature, so that the steel strip indented a groove in the initial aluminum strip (Fig. 1b). The depth of the groove and the respective local strip thickness h1.0 (s. Fig. 1b) depends on the number of rolling passes as well as on the set of roll gaps. However, due to excessive reduction at a single rolling pass, the defects of the aluminum strips such as buckles or cracks occur. This limitation caused the application of multi-pass rolling with relatively low deformation strains of single passes. On the basis of the latter behavior, the five pre-profiled strips with different groove depth as well as three pre-profiled strips with the same groove depth were prepared.

Afterward, all of the pre-profiled aluminum strips were subjected to a heat treatment for the stress relief which is obtained during the cold rolling of metal. As the mode of heat treatment, the recrystallization annealing in a resistance furnace at temperature of $375^{\circ} \mathrm{C}$ with the soaking duration of 1 hour and a subsequent free air cooling was chosen. The mechanical properties of the material after the heat treatment measured in accordance with DIN EN ISO 6892-1: 2009 for tensile tests and ISO 6506-1: 2014 for Brinell hardness tests are listed in Table 2. The different mechanical properties for various conditions are caused by different recrystallization behaviors of the deformed and non-deformed material during the annealing. 
Table 2. Mechanical properties of the aluminum alloy EN AW-1050 after the heat treatment

\begin{tabular}{|c|c|c|c|c|c|c|c|}
\hline State & Thickness & \multicolumn{2}{|c|}{ Yield stress [MPa] } & \multicolumn{2}{c|}{ Tensile strength [MPa] } & \multicolumn{2}{c|}{ Hardness, HBW 2,5/31,25 } \\
\cline { 3 - 8 } & {$[\mathrm{mm}]$} & $\min$. & $\max$ & $\min$. & $\max$. & $\min$. & max. \\
\hline $\mathrm{O}$ & $2.0-2.9$ & 63.5 & 89.0 & 80.0 & 100.1 & 24 & 29 \\
\hline
\end{tabular}

After the stress relieving heat treatment, the pre-profiled strips have been flat rolled in one pass at room temperature in the same rolling direction. As result of this, all of the aluminum strips possess a constant thickness h2 (Fig. 1c). Due to the different deformation hardening of the preprofiled regions of the strips, tailored mechanical properties across their width were generated.

In the first series of experiments, five samples with grooves of varying depth were subjected to rolling employing a reduction corresponding to the thickness difference between the pre-profiled strip elements $\Delta \mathrm{h}=\mathrm{h} 1.1$ - h1.0. In accordance with this condition, solely thicker regions of the strip were deformed. The aim of this study was to show how the differences in the reduction of various strip elements affect the tailoring of mechanical properties.

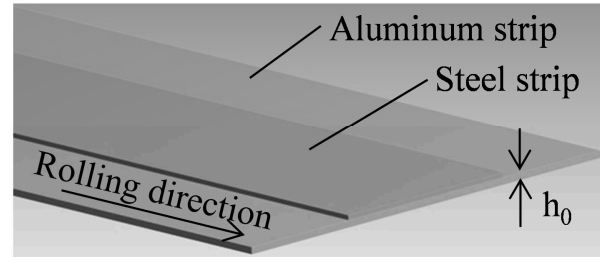

a)

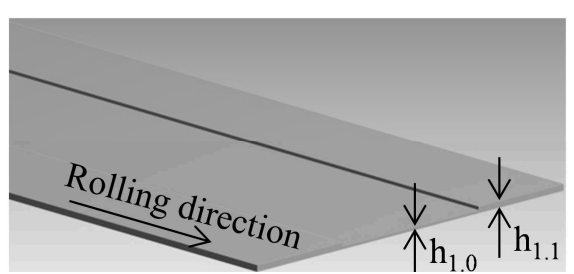

b)

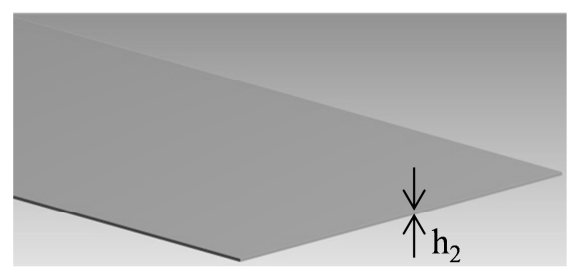

c)

Fig. 1. Schematic representation of the experimental procedure: a) sample prepared for the first flat rolling pass, b) pre-profiled sample after the several flat rolling passes, c) flat strip with tailored across the width mechanical properties

In the second series of tests, three samples with the same cross section shape were rolled using varying strains. Correspondingly, the reduction difference $\Delta \mathrm{h}$ for these pre-profiled strips elements was equal. Thus, it should be investigated how the relationship of the deformation strains of diverse strip zones effect on the mechanical properties heterogeneity.

The rolling parameters of the all pre-profiled and annealed strips are listed in Table 3.

Table 3. Parameters of flat rolling of the pre-profiled aluminum strips

\begin{tabular}{|c|c|c|c|c|c|c|}
\hline \multirow{2}{*}{$\begin{array}{c}\text { No } \\
\cdot\end{array}$} & \multicolumn{2}{|c|}{ Initial size } & After rolling & \multirow{2}{*}{$\varphi_{1}$} & \multirow{2}{*}{$\varphi_{2}$} & \multirow{2}{*}{$\Delta \varphi$} \\
\cline { 2 - 5 } & $\mathrm{h}_{1.0}[\mathrm{~mm}]$ & $\mathrm{h}_{1.1}[\mathrm{~mm}]$ & $\mathrm{h}_{2}[\mathrm{~mm}]$ & \multicolumn{7}{|c|}{ First series of experiments } \\
\hline 1 & 2.8 & 2.9 & 2.602 & 0.073 & 0.108 & 0.035 \\
\hline 2 & 2.5 & 2.9 & 2.459 & 0.016 & 0.165 & 0.149 \\
\hline 3 & 2.3 & 2.9 & 2.294 & 0.002 & 0.234 & 0.232 \\
\hline 4 & 2.1 & 2.9 & 2.093 & 0.003 & 0.326 & 0.323 \\
\hline 5 & 2.0 & 2.9 & 1.989 & 0.005 & 0.377 & 0.372 \\
\hline \multicolumn{7}{|c|}{ Second series of experiments } \\
\hline 6 & 2.1 & 2.65 & 2.1 & 0 & 0.232 & 0.232 \\
\hline 7 & 2.1 & 2.65 & 2.02 & 0.039 & 0.271 & 0.232 \\
\hline 8 & 2.1 & 2.65 & 1.7 & 0.211 & 0.443 & 0.232 \\
\hline
\end{tabular}


All of the rolling experiments were realized by means of a two high roll stand of the Chair of Materials Science of the University of Paderborn. The rolls diameter of the applied machine is $210 \mathrm{~mm}$. The barrel length amounts to $300 \mathrm{~mm}$. The roll stand is equipped with two electric motors of the total power of $22 \mathrm{~kW}$. Before a rolling procedure, the stand is pre-stressed by means of a hydraulic system. Thus, the springing of the machine is significantly reduced. The used for experiments two high roll stand is depicted in Fig. 2.

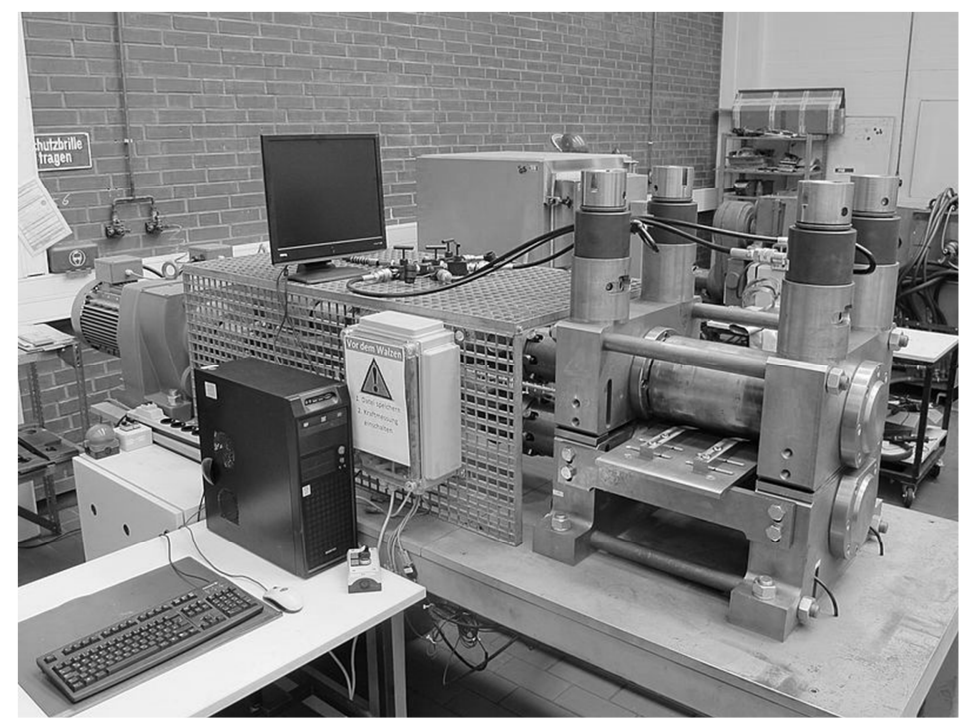

Fig. 2. Two high roll stand of the Chair of Materials Science, University of Paderborn

After the rolling of pre-profiled strips to the flat products of a constant thickness, they were subjected to mechanical tests as well as to microstructure analysis. For this purpose, the tensile test specimens from the diverse strain hardened regions of the strips were sampled.

The samples orientation corresponded to the rolling direction. Subsequently Brinell hardness tests on the rolled flat strips were performed. Comparative results of the tensile tests and hardness tests are summarized in Fig. 3 for the first series of experiments and in Fig. 4 for the second series of investigation. The mechanical properties characteristics of the strip areas, which were subjected to a less deformation, are labeled as $\mathrm{R}_{\mathrm{p} 0.2,} \mathrm{R}_{\mathrm{m}}{ }_{\mathrm{m}}$ and $\mathrm{HBW}{ }^{\prime}$. The characteristics of the major strained strip regions are named $\mathrm{R}_{\mathrm{p} 0.2}, \mathrm{R}_{\mathrm{m}}$ and $\mathrm{HBW}$.

\section{Results}

The outcomes in Fig. 3 display that the major effect of tailored strain hardening can be observed by true strain value of the thicker pre-profiled strip region with approximately $\varphi_{2}=0.165$. Furthermore, the yield stress possesses an increased sensitiveness to various deformation conditions compared to the tensile strengths. The averaged yield stress ratios i.e., $\mathrm{R}_{\mathrm{p} 0.2} / \mathrm{R}_{\mathrm{p} 0.2}^{\prime}$ exhibit a strain value of $28 \%$. The maximum measured distinction of the yield stress for one strip rises up to $35 \%$. An increased deformation heterogeneity can be detected for $\Delta \varphi=0.372$ which leads to a stabilizing of yield stress, tensile strength and hardness ratios at $20 \%$. The maximum hardness distinction was $40 \%$ which corresponds to the true strain value of $\varphi_{2}=0.323$.

The second experiment set (See Fig. 4) shows that the simultaneous deformation of both preprofiled strip areas leads to reduced tailored properties. These property ratios steadily decrease with higher reductions of the thinner strip section. At a deformation strain of $\varphi_{1}=0.33$, tailored strength properties are not more observed. Moreover, a difference in hardness of approximately $18 \%$ can be detected. But it is also reduced with an increasing total strain.

In addition, specimens from different strip regions for metallographic examinations were cut by electric discharge machining. The preparation of the micro-sections included grinding, polishing and electrolytic etching according to Barker. The microstructure of the strip region, which was barely subjected to plastic deformation, as well as the microstructure of the area, which was 
significant strained, are depicted in Fig. 5a and in Fig. 5b respectively. The grains in the rarely deformed metal zone are coarser. It can be caused by recrystallization and subsequent grain growth in the pre-profiled strip zone during the preliminary annealing. The finer microstructure of the strained strip area consists of grains, which are elongated in the rolling direction. This is typical for materials processed by means of cold rolling. The differences of the microstructure of the various strained aluminum strip regions accord with the observed mechanical properties distribution across the strip width.

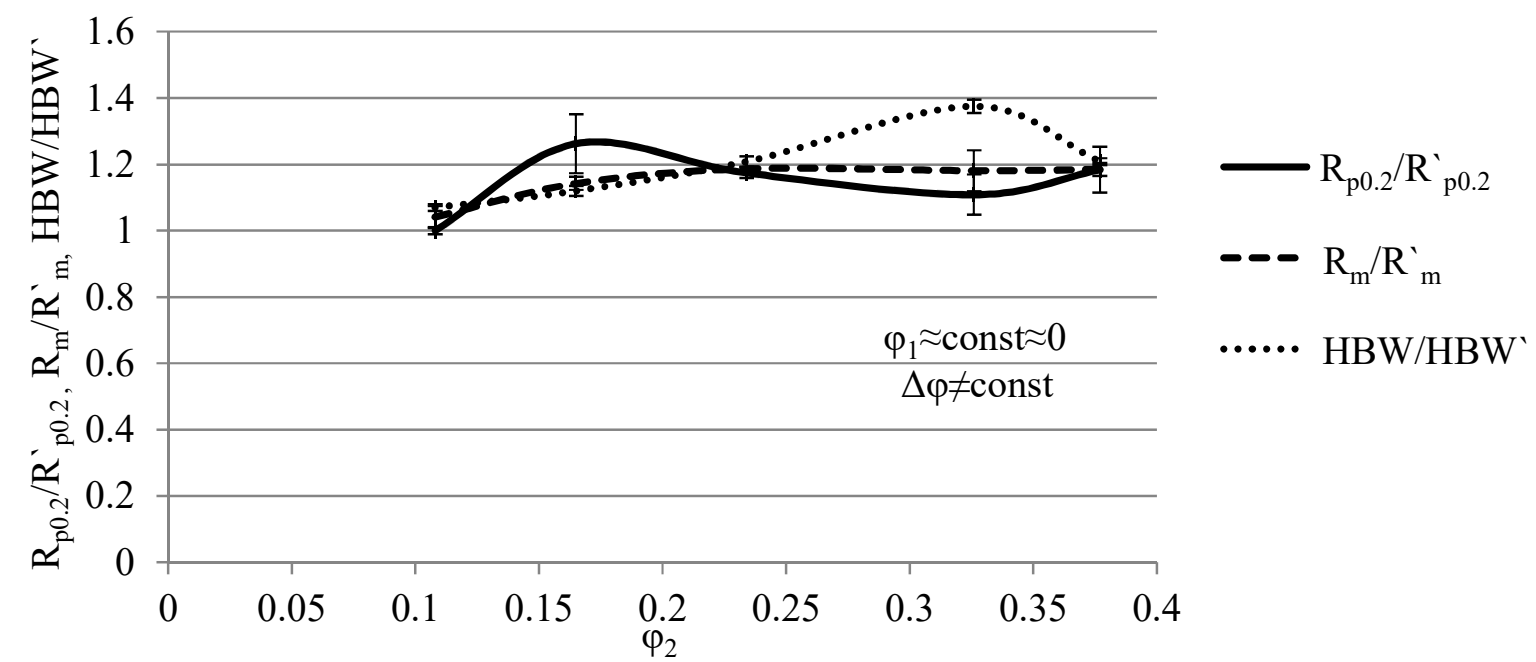

Fig. 3. Comparison of mechanical properties in different strained strip areas for the condition

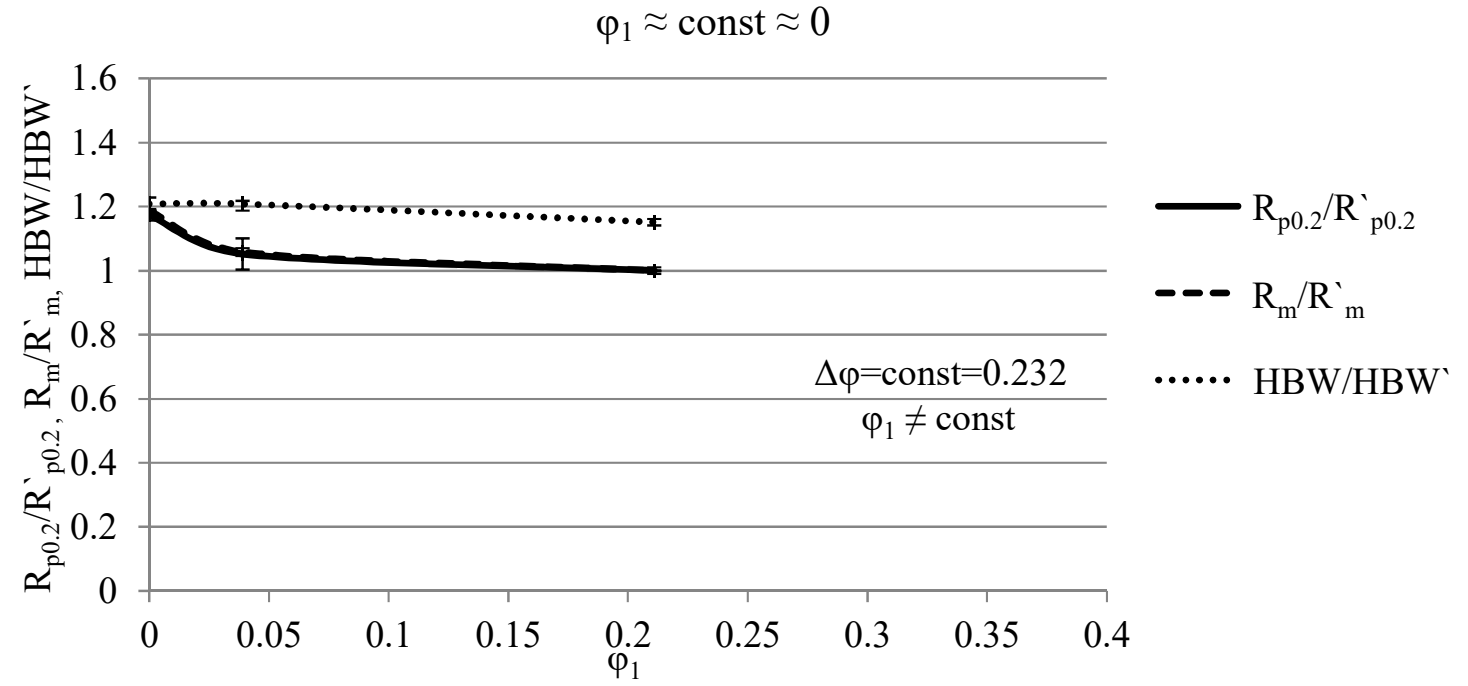

Fig. 4. Comparison of mechanical properties in different strained strip areas for the condition $\Delta \varphi=$ const $=0.232$

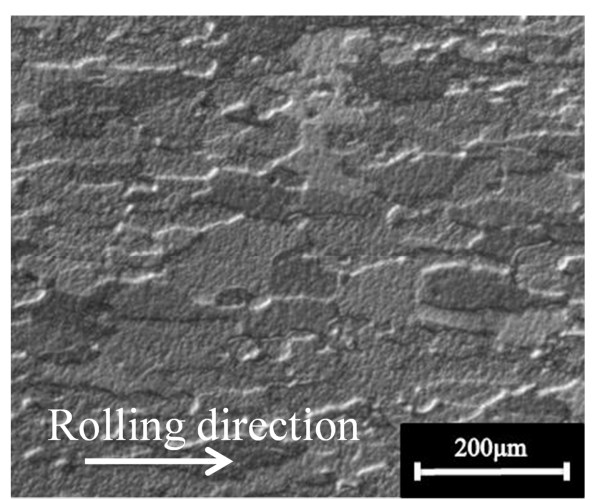

a)

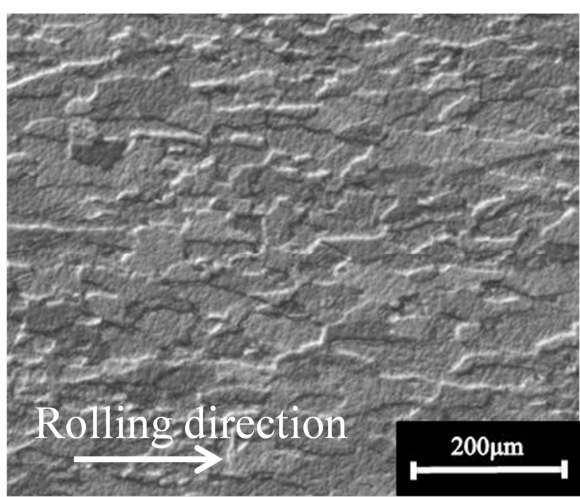

b)

Fig. 5. Microstructure of different regions of a strip, which was reduced with the strain $\varphi_{1}=0.002$ (a) and with the strain $\varphi_{2}=0.232$ (b) 


\section{Conclusions and Perspectives}

The experimental investigation on the cold flat rolling of pre-profiled strips of aluminum alloy EN AW-1050 proves the possibility to induce tailored mechanical properties due to different strain hardening of various strip regions. The major variation between the mechanical characteristics of different strip's elements is found in the hardness and reaches $40 \%$. The maximum distinction of yield stress amounts $35 \%$. This corresponds to the deformation of the thick strip sections with a true strain of 0.165 . In consequence with the latter requirement, a deformation of the thin preprofiled strip element has to be avoided. A further increasing of the thick strip region reduction without deformation of the thin strip area at once stabilizes the yield and tensile strength distinction on a level of about $20 \%$. A deformation of both areas of pre-profiled strips causes a decreasing of the effect of tailored properties. At a deformation strain of 0.211 for the thin element as well as 0.443 for the thick region of pre-profiled strip the strength properties in the rolling direction are homogeneous.

Outside the scope of investigations remains the problem of residual stresses evolution. It can be critical for the flat rolling of pre-profiled strips even if the quality of their shape is good. An evolution of stress state of material during the rolling will be analyzed by the means of numerical simulation in further studies.

Another critical issue is the preparation of the pre-profiled strip. The procedure utilized in the scope of the experiments shows a lavishness of the multi-pass rolling of aluminum-steel sandwiches. An alternative method for the pre-profiled strips manufacturing provides a twin-roll casting. This was already applied successfully on an example of steel strips with profiled cross section by M. Vidoni, G. Hirt et al. [8].

\section{References}

[1] R.J. Pallet, R.J. Lark, The use of tailored blanks in the manufacture of construction components, JOMPT 117 (2001) 249-254.

[2] R. Perez-Santiago, E. Billur, A. Ademaj, C. Sarmiento, R. Berlanga, T. Altan, Hot stamping a B-pillar with tailored properties: experiments and preliminary simulation results, Proc. 4th Int. Hot Stamping Conference, Lulea, Sweden (2013) 83-90.

[3] M. Merklein, M. Johannes, M. Lechner, A. Kuppert, A review on tailored blanks - Production, applications and evaluation, JOMPT 214 (2014) 151-164.

[4] M. Merklein, M. Lechner, Manufacturing flexibilisation of metal forming components by tailored blanks, Proc. Conf. Competitive Manufacturing 2013 165-170.

[5] G. Hirt, D.H. Davalos-Julca, Tailored profiles made of tailor rolled strips by roll forming - part 1 of 2, Steel Research int. 83 (2012) 100-105.

[6] R. Kopp (1), P. Böhlke, A new rolling process for strips with a defined cross section, CIRP Annals Manufacturing Technology 52, Issue 1 (2003) 197-200.

[7] M. Geiger, M. Merklein, U. Vogt, Aluminum tailored heat treated blanks, Prod. Eng. Res. Devel. 3 (2009) 401-410.

[8] M. Vidoni, M. Daamen, G. Hirt, Advances in the twin-roll strip casting of strips with profiled cross section, Key Eng. Mat. 554-557 (2013) 562-571. 